\title{
A New Planning Method to Easily Harvest the Superficial Circumflex Iliac Artery Perforator Flap
}

\author{
Nicolás Pereira, MD, MSc ${ }^{1,2}$ Leonardo Parada, MD, MHA ${ }^{1}$ Matías Kufeke, MD ${ }^{1,2}$ \\ Ekaterina Troncoso, MD ${ }^{1}$ Ricardo Roa, MD ${ }^{1}$
}

${ }^{1}$ Department of Plastic Surgery and Burns, Hospital del Trabajador, Santiago, Chile

${ }^{2}$ Department of Plastic Surgery, Clínica Las Condes, Santiago, Chile

Address for correspondence Nicolás Pereira, MD, MSc, Department of Plastic Surgery and Burns, Hospital del Trabajador, 185 Ramon Carnicer, 5th Floor, Providencia 7501239, Santiago, Chile

J Reconstr Microsurg 2020;36:165-170.

(e-mail: npereira@hts.cl).

\begin{abstract}
Keywords

- superficial circumflex iliac artery perforator flap

- preoperative imaging

- planning method

Background The superficial circumflex iliac artery perforator flap (SCIP) is a thin, pliable, and versatile flap used mainly for extremities and head and neck reconstruction. Different planning methods have been described, but these are not yet standardized like in other flaps. The aim of this study is to present a fast, effective, and reliable method for SCIP flap planning using computed tomography angiography (CTA).

Patients and Methods Between October 2017 and September 2018, CTA was performed on 40 patients. Preoperative planning of SCIP flaps based on the medial branch was performed analyzing CTA images. The perforating sites of the medial branch on the deep (point D) and superficial fascia (point S) were identified. Distances to those points, from the center of the umbilicus in the " $y$-axis" and the midline perpendicularly in the " $x$-axis," were measured. These measurements were transferred to the patient's skin as a guide for dissection.

Results Eighty areas were studied identifying points D and S in CTA. Forty-three SCIP flaps were performed using this planning method. In $100 \%$ of the flaps, points D and $S$ matched perfectly with handheld Doppler and surgical findings.

Conclusion Points D and S method for medial branch based SCIP planning with CTA is an easy to learn, efficient, fast, and reliable technique for preoperative planning, allowing a safe and predictable elevation of the flap.
\end{abstract}

The inguinal area has been used as a donor site since a long time ago. Initially, McGregor described the pedicled inguinal flap in $1972^{1}$ which later become the first free flap performed by Daniel and Taylor. ${ }^{2}$ However, given the frequent anatomical variations of its vasculature, it did not achieve great popularity as a free flap. Afterward, in the perforator flaps era, the inguinal area reappeared as the superficial circumflex iliac artery perforator flap (SCIP). Since its description by Koshima et al in 2004, ${ }^{3}$ it has been used in head and neck, ${ }^{4,5}$ external auditory canal, ${ }^{6}$ penis, ${ }^{7}$ scrotum $^{8}$, vulvar $^{9}$, groin ${ }^{10}$, bone, ${ }^{11}$ and lower limb reconstruction. ${ }^{12,13}$

The SCIP flap is based on perforators dependent on the superficial circumflex iliac artery that originates from the

received

May 21, 2019

accepted after revision

August 27, 2019

published online

October 21, 2019 femoral artery vessels in most of the cases. This flap is generally thin and pliable, of moderate size, and with a concealed donor site. Their limitations include a restricted width to allow a primary closure, increased thickness in overweight patients, and short pedicle with low caliber which restricts its use to defects with nearby recipient vessels. To face these disadvantages, modifications, such as those introduced by Hong et al, ${ }^{13}$ with dissection above the superficial fascia based on the media branch, and the use of supermicrosurgical techniques for perforator-to-perforator anastomosis have expanded its uses.

As with other free flaps, ${ }^{14}$ preoperative planning has been described by different authors using hand-held Doppler, color Doppler ultrasound (CDU), ${ }^{15-17}$ angiography, and
Copyright $\odot 2020$ by Thieme Medical Publishers, Inc., 333 Seventh Avenue, New York, NY 10001, USA. Tel: +1(212) 760-0888.
DOI https://doi.org/ 10.1055/s-0039-1698444. ISSN 0743-684X. 
computed tomography angiography (CTA). ${ }^{13,18}$ The wide majority describes the use of CTA and CDU. ${ }^{19}$ However, these reports are mostly limited to point out the use of such technologies without making a complete description of the way in which the information is used to plan the flap.

Despite the changes introduced, the better anatomical knowledge and the varieties of methods proposed to study its vasculature; SCIP flap is not widely used in western countries or by novice microsurgeons. Its planning and marking based on CTA have not been standardized like other flaps, such as anterolateral thigh (ALT) flap.

We have noticed that after penetrating the deep fascia, the medial perforator generally provides some branches to the inguinal lymph nodes before penetrating the superficial fascia. Therefore, the perforating site of the medial branch on the deep fascia is generally different from the perforating site on the superficial fascia. Considering this variability and the absence of a preoperative standardization, the aim of this study is to present an effective and reliable method to plan the medial branch based SCIP free flap using CTA, allowing an efficient, fast, and low dependent on the operator technique, even for novice surgeons.

\section{Patients and Methods}

Microsurgical reconstructions with the medial branch based SCIP free flap were planned using CTA and performed by the same team (N.P. and M.K.), in the Department of Plastic Surgery and Burns of Hospital del Trabajador and Department of Plastic Surgery of Clínica Las Condes, between January and December 2018.

After signing a written consent authorizing the study, lower extremity CTA images were obtained using a 64-detector row helical CT system (Brilliance 64; Philips, Amsterdam, the Netherlands) with perforator protocol, maximum intensity projection, and 1-mm slice width; to study recipient vessels and for free flap preoperative planning.

CTA images were analyzed by the surgeon preoperatively using XERO Viewer 8.1.1 (Agfa HealthCare; Mortsel, Belgium). Different measurements were performed in both inguinal areas, considering the midline as "y-axis" (1-mm slice width) and a perpendicular line to the latter as the "x-axis."

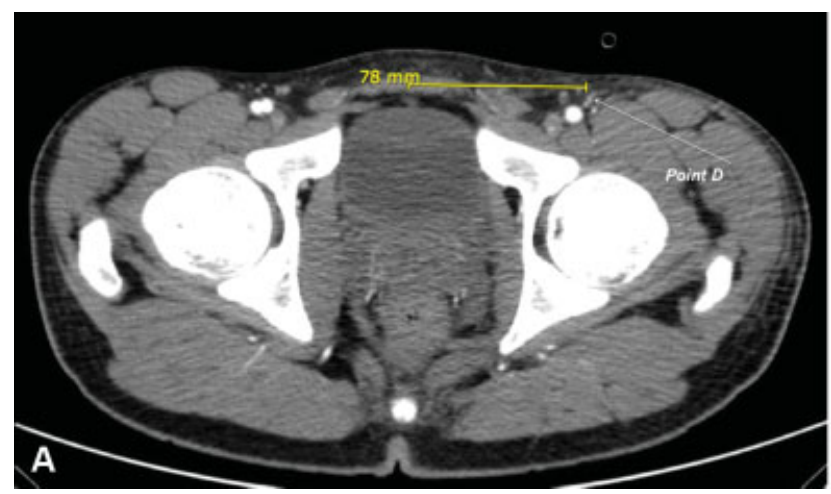

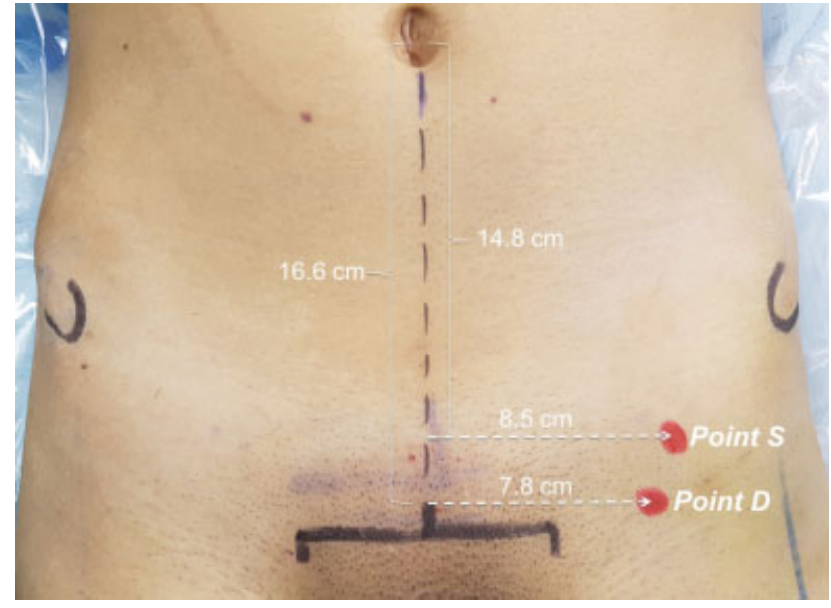

Fig. 2 Points $D$ and $S$ were marked on the patient's skin of the inguinal area preoperatively (same patient as in - Fig. 1).

- Step 1: in the axial view, we studied the course of the superficial circumflex iliac artery (SCIA), from its origin on the femoral artery (most of the cases). We identified the perforating site of the medial branch on the deep fascia. Distances were measured from the center of the umbilicus in the "y-axis" and from the midline perpendicularly in the "X-axis." We named this site as "point D" (deep fascia; - Fig. 1A).

- Step 2: in the axial view, we identified the perforating site of the medial branch on the superficial fascia, usually when it separates from the lymph nodes. Measurements were taken from the center of the umbilicus in the "y-axis" and from the midline perpendicularly in the " $\mathrm{x}$-axis." We named this site as "point S" (superficial/Scarpa's fascia; - Fig. 1B).

Points D and S were marked on the patient's skin of both inguinal areas preoperatively (-Fig. 2). The hand-held Doppler ultrasound (8-MHz probe) was performed to check the presence of the perforator on the marked points.

SCIP flaps were designed with the axis centered in a line from the inguinal crease to the anterior superior iliac spine (ASIS) as described by Goh et al. ${ }^{20}$ An exploratory incision was performed in the inferior-lateral aspect and the flap was

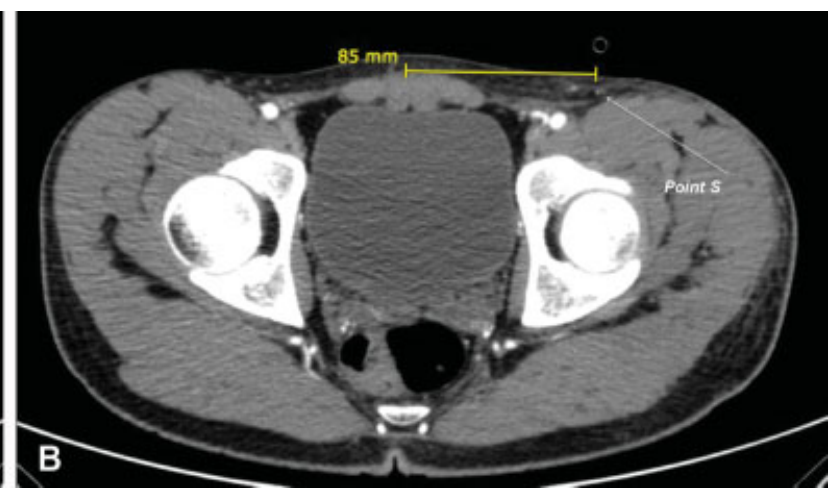

Fig. 1 Identification of perforating sites of the medial branch of the superficial circumflex iliac artery on CTA. The perforating site on the deep fascia was named as "Point D" (A) and on the superficial fascia as "Point S" (B). Distances were measured from the center of the umbilicus in the "y-axis" (not shown) and from the midline perpendicularly to the latter, in the "x-axis" (yellow line). 
raised above the superficial fascia up to 1 to $2 \mathrm{~cm}$ from point $D$, where we deepened to look for the perforator on its origin. A vessels loop was placed once it was identified. Dissection continued above the superficial fascia up to point $S$, which we used as a reference for the raising plane of the flap to include the pedicle on it. The perforating sites of the medial branch of SCIA on the deep and superficial fascia were correlated with preoperative markings (points D and S).

The relation between the distance from points $D$ to $S$ (distance DS) and the body mass index (BMI) was studied. Before analysis, data were tested for normality using Kolmogorove-Smirnov test. Descriptive characteristics were presented as mean and range. The relation between distance DS and BMI was performed with the Pearson's correlation coefficient, using SPSS 25.0 (IBM Corp., Amornk, NY). Statistical significance was set at $p<0.05$.

\section{Results}

In 40 consecutive patients, candidates for lower or upper extremity reconstruction, 80 inguinal areas were analyzed in CTAs. Thirty-two men and eight female were included. Mean body mass index (BMI) was $27.2 \mathrm{~kg} / \mathrm{m}^{2}$ (range: $20-$ $\left.35 \mathrm{~kg} / \mathrm{m}^{2}\right)$; normal BMI $\left(<25 \mathrm{~kg} / \mathrm{m}^{2}\right)$ was presented in $29.7 \%$, overweight (range: $25-30 \mathrm{~kg} / \mathrm{m}^{2}$ ) in $37.8 \%$ and obesity $\left(>30 \mathrm{~kg} / \mathrm{m}^{2}\right)$ in $32.5 \%$ of the cases. Forty-three SCIP free flaps were performed for upper and lower extremities reconstruction (three double SCIP flap cases).

The identification of points $\mathrm{D}$ and $\mathrm{S}$ in CTA was performed in all 80 inguinal areas and points were marked on the patient's skin preoperatively. Mean distance from midline (x-axis) to point D was $7.41 \mathrm{~cm}$ (range: $5.9-9.3 \mathrm{~cm}$ ) and to point $S$ was $9.11 \mathrm{~cm}$ (range: $6.9-13 \mathrm{~cm}$ ). Mean distance from point D to point $S$ (distance DS) was $1.7 \mathrm{~cm}$ (range: $0-4.7 \mathrm{~cm}$ ). Distance DS tended to be greater when BMI was higher. Significant difference in distance DS was found between patients with $\mathrm{BMI}<28 \mathrm{~kg} / \mathrm{m}^{2}$ and $\geq 28 \mathrm{~kg} / \mathrm{m}^{2} \quad(p=0.08$; - Table 1). The Pearson's correlation coefficient between distance DS and BMI was 0.265 with a $p$-value $=0.023$. The hand-held Doppler was performed in all 160 markings (80 points $\mathrm{D}$ and 80 points $\mathrm{S}$ ) and signals were detected in $100 \%$ of the cases ( - Video 1 Identification of points D and S in CTA, markings and correlation with the hand-held Doppler).

Table 1 Distances from midline to points D and S, distance DS, and distance DS depending on the BMI

\begin{tabular}{|l|l|l|}
\hline & Mean $(\mathrm{cm})$ & Range $(\mathrm{cm})$ \\
\hline Midline to point D & 7.41 & $5.9-9.3$ \\
\hline Midline to point S & 9.11 & $6.9-13$ \\
\hline Distance DS (general) & 1.7 & $0-4.7$ \\
\hline Distance DS $(\mathrm{BMI}<28)$ & 1.49 & $0.4-3.9$ \\
\hline Distance DS $(\mathrm{BMI} \geq 28)$ & 2.07 & $0-4.7$ \\
\hline
\end{tabular}

Abbreviations: BMI, body mass index; point D, perforating site of the medial branch on the deep fascia; point $S$, perforating site of the medial branch on the superficial fascia; DS, distance between points $D$ and S.

\section{Video 1}

Identification of points D and S in CTA, markings, and correlation with hand-held Doppler. CTA, computed tomography angiography; point $\mathrm{D}$, perforating site of the medial branch on the deep fascia; point $S$, perforating site of the medial branch on the superficial fascia. Online content including video sequences viewable at: https://www.thieme-connect.com/ products/ejournals/10.1055/s-0039-1698444.

Forty-three SCIP free flaps were raised above the superficial fascia and intraoperative identification of points $\mathrm{D}$ and $\mathrm{S}$ was performed. Preoperative markings and intraoperative finding coincided in all 86 points ( 43 points $D$ and 43 points S; $100 \%$ of the cases; - Video 2, Preoperative marking of points $\mathrm{D}$ and $\mathrm{S}$ and correlation with intraoperative findings).

\section{Video 2 \\ Preoperative marking of points $\mathrm{D}$ and $\mathrm{S}$ and correlation with intraoperative findings. Point $\mathrm{D}$, perforating site of the medial branch on the deep fascia; point S, perforating site of the medial branch on the superficial fascia. Online content including video sequences viewable at: https://www.thieme-connect.com/ products/ejournals/10.1055/s-0039-1698444.}

\section{Discussion}

SCIP flap planning using points $\mathrm{D}$ and $\mathrm{S}$ method based on CTA findings allows an effective and reliable approach even for novice surgeons. Ii is an efficient, fast, and low dependent on the operator technique that allows an adequate planning for performing the flap.

Since the description by Koshima et al in $2004^{3}$ and subsequent modifications, ${ }^{13,21}$ the use of the SCIP flap has expanded to different fields, mainly head and neck, 4,5,22,23 and lower extremity reconstruction. $3,12,13,20,22,24$ It is raised on perforators from the superficial circumflex iliac artery, which in $83.5 \%$ originates from the femoral artery, the superficial femoral artery in $8.5 \%$, the deep femoral artery in $7 \%$, and infrequently in the lateral circumflex femoral artery (1\%). ${ }^{18} \mathrm{He}$ et al ${ }^{19}$ describe different relations of the SCIA with surrounding vessels, originating in a short or longer common trunk with the deep circumflex iliac artery, both separately from the femoral artery, or sharing a common trunk with the superficial inferior epigastric artery (SIEA). The SCIA has two perforators, one medial (superficial) and one lateral (deep).

It has been described two distribution patterns of the medial branch, after penetrating the deep and superficial fascia. In $44 \%$ has an axial pattern with a course toward the anterior superior iliac spine and in $56 \%$ has a short perforator 


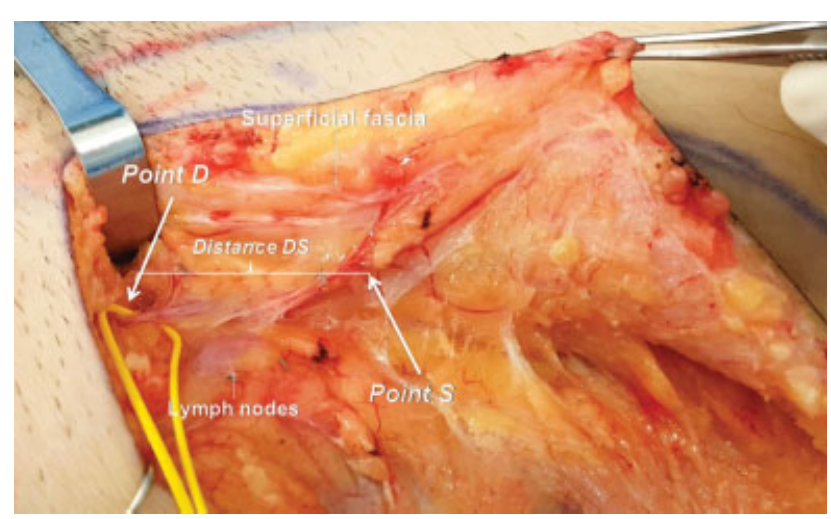

Fig. 3 Perforating sites of the medial branch of SCIA on the deep (point D) and superficial (point S) fascia. SCIA, superficial circumflex iliac artery.

directly anchored to the dermis. ${ }^{18}$ The use of the medial perforator allows obtaining thinner flaps with a faster elevation, since it avoids intramuscular dissection and limits the dissection under the deep fascia only to the most proximal area to obtain a longer pedicle. The superficial planes of elevation may reduce the risk of lymphatic injuries and postoperative lymphoceles or lymphorrea. ${ }^{25}$ In different series, this perforator is used and is preferably chosen by the authors. We have noticed that after penetrating the deep fascia, the medial perforator has a course within the deep-fat layer and generally provides some branches to the inguinal lymph nodes before penetrating the superficial fascia; afterward it travels through the superficial fat layer toward the anterior superior iliac spine. Thus, the perforating site level of the medial branch on the deep fascia with respect to the midline is different from the perforating site on the superficial fascia (-Fig. 3 ).

Our observations differ from those described by Suh et al, ${ }^{18}$ who reported that after penetrating the deep fascia, the medial perforator immediately enters the superficial fascia. The difference lies mainly in the physical characteristics of the patients, being the mean BMI $23.8 \mathrm{~kg} / \mathrm{m}^{2}$ in a Korean study ${ }^{20}$ and $27.2 \mathrm{~kg} / \mathrm{m}^{2}$ in this series. In Eastern countries, patients tend to be skinny; therefore, the thickness of the flaps is thinner. In Western population, the prevalence of overweight and obesity is higher which is why our series has a higher proportion of patients with this condition (70\% with overweight or obesity) that represents our population. In our series, distance DS tended to be greater when BMI was higher, with a positive correlation between both measures. Probably, in the presence of a thicker deep-fat layer, a longer pathway of the medial branch exists, so points $D$ and $S$ method becomes more important for predicting the course and planning the flap.

As with other perforator flaps, preoperative planning is of great importance, ${ }^{14,26}$ allowing to know the location and the course of the vessels to choose the most appropriate perforator, raising the flap in a safe way. This fact is even more important in SCIP flap given the wide anatomical variations described in the literature which is generally the reason attributed to its less frequent use than other perforator flaps. Recently, Gentileschi et $\mathrm{al}^{27}$ performed a surgical and radio- anatomical study analyzing high-resolution contrast-enhanced CT, with particular attention to the deep branch (DB) of the SCIA. They employed a system of coordinates based on the line between pubic tubercle (PT) and anterior superior iliac spine (ASIS) to describe the position of the perforator of the DB. Twenty-three patients were also studied by detecting the surgical anatomy during SCIP flap harvest, finding a 100\% correlation between surgical and radiological findings. Points $\mathrm{D}$ and $\mathrm{S}$ method uses the center of the umbilicus and the midline as references for measurements. We consider that using superficial anatomical landmarks (umbilicus) for preoperative planning is more accurate since in some patients (overweight or obese), bony landmarks, such as PT or pubic symphysis, are difficult to palpate and to transfer the level of deep structures to the surface of the skin may be inaccurate. We also had a $100 \%$ of correlation with intraoperative findings in all 43 cases operated, but we prefer to base the SCIP flap on the superficial branch since it allows thinner flaps and a lower potential risk of lymphatic problems.

CTA is widely available and is a quick imaging exam with few complications, generally useful for preoperative study of the underlying disease (trauma, tumor, recipient vessels availability, etc.) of patients who require free flap reconstruction. It allows knowing in great detail the vascular anatomy of the inguinal area, including origin and branching pattern of SCIA and its perforators. Other nearby vessels, such as the superficial inferior epigastric artery and superficial external pudendal artery, can be studied as well. In addition, the caliber and relations between these vessels can be evaluated, selecting the most suitable for the flap.

Using our proposed method, we analyzed 160 perforating sites in 80 inguinal areas; points $D$ and $S$ were identified and marked on the skin surface in all cases. We raised 43 SCIP free flaps with $100 \%$ of concordance between the predicted anatomy of the perforating sites by CTA and surgical findings. In this way, we can point out the following advantages of our method as follows:

1. Perforator vessels analysis on CTA can be performed by the surgeon, without needing radiologists report. The anatomy of the vascular, lymphatic, and muscular structures of the inguinal area can be evaluated, facilitating the subsequent dissection.

2. Identifying points $D$ and $S$ provide more information and aids, more predictable dissection throughout the surgery, considering the course of the medial perforator at different levels.

3. The use of superficial anatomical landmarks is more accurate, especially in patients with excess weight because the information is transferred directly to the skin to be used as a guide during surgery.

4. Relating the medial branch in CTA with recognizable superficial anatomical landmarks on the skin allows tracing the pedicle. In this way, the surgeon can predict with accurately intraoperative findings and elevate the flap safer and faster.

5. With all this information, it is not strictly necessary to perform a complementary Doppler ultrasound. 
Some of the limitations of this method include the following:

1. Recognizing the perforator vessel can be difficult in some cases, especially in short and/or small caliber pedicle.

2. CTA uses intravenous contrast and has associated ionizing radiation risk.

3. CTA does not provide functional information about the vessels flow.

The most frequently planning methods reported are color Doppler ultrasound and CTA. Regarding color Doppler, its advantages lie on the wide availability of the exam; on expert hands is quick to perform; it does not involve radiation or the use of contrast and provides hemodynamic information of the vessels, being able to detect the anchoring point to the dermis, thus facilitating the harvesting of thin and ultrathin flaps. ${ }^{28}$ On the other hand, its disadvantages are the ability to detect only vessels larger than $0.5 \mathrm{~mm}$, does not provide a three-dimensional (3D) description of the anatomy, to be an operator-dependent test, and it is not easy to perform and to interpret. $^{29,30}$

CTA advantages are its ability to capture vessels of smaller caliber than ultrasound, larger than $0.3 \mathrm{~mm}$. It provides 3D information of the vessels and their surrounding tissues, allowing even 3D reconstructions that can be interpreted easier. Moreover, these 3D reconstructions can be used by augmented reality and predict the complete path of the pedicle to the skin. ${ }^{31}$ Its disadvantages are the need of intravenous contrast and radiation, not providing hemodynamic information. ${ }^{29-33}$

Considering all the above, the majority of studies on SCIP flap planning propose the use of both technologies together. ${ }^{19}$ However, based on our experience, points D and S method with CTA may be enough for a successful SCIP flap preoperative plan.

We have demonstrated that this planning method based on CTA is reliable and efficient. A brief analysis allows any microsurgeon to easily plan a SCIP flap and transfer all this information to the patient, safely raising the flap. We hope that future refinements to the method allow better planning and to add this flap as a workhorse of any reconstructive plastic surgeon.

\section{Conclusion}

Medial branch based SCIP flap planning with CTA using the points $\mathrm{D}$ and $\mathrm{S}$ method is an easy to learn, efficient, fast, and reliable technique that allows performing a safe and predictable raise of the flap, even for novice surgeons. It is especially useful in overweight and obese patients where the DS distance tends to be higher.

\section{Funding}

None.

Conflict of Interest

None declared.

\section{References}

1 McGregor IA, Jackson IT. The groin flap. Br J Plast Surg 1972;25 (01):3-16

2 Daniel RK, Taylor GI. Distant transfer of an island flap by microvascular anastomoses. A clinical technique. Plast Reconstr Surg 1973;52(02):111-117

3 Koshima I, Nanba Y, Tsutsui T, et al. Superficial circumflex iliac artery perforator flap for reconstruction of limb defects. Plast Reconstr Surg 2004;113(01):233-240

4 Iida T, Mihara M, Yoshimatsu H, Narushima M, Koshima I. Versatility of the superficial circumflex iliac artery perforator flap in head and neck reconstruction. Ann Plast Surg 2014;72(03): 332-336

5 Iida T, Narushima M, Yoshimatsu H, Yamamoto T, Araki J, Koshima I. A free vascularised iliac bone flap based on superficial circumflex iliac perforators for head and neck reconstruction. J Plast Reconstr Aesthet Surg 2013;66(11):1596-1599

6 Iida T, Mihara M, Yoshimatsu H, Narushima M, Koshima I. Reconstruction of the external auditory canal using a superthin superficial circumflex iliac perforator flap after tumour resection. J Plast Reconstr Aesthet Surg 2013;66(03):430-433

7 Koshima I, Nanba Y, Nagai A, Nakatsuka M, Sato T, Kuroda S. Penile reconstruction with bilateral superficial circumflex iliac artery perforator (SCIP) flaps. J Reconstr Microsurg 2006;22(03):137-142

8 Han HH, Lee JH, Kim SM, Jun YJ, Kim YJ. Scrotal reconstruction using a superficial circumflex iliac artery perforator flap following Fournier's gangrene. Int Wound J 2016;13(05):996-999

9 Gentileschi S, Servillo M, Garganese G, et al. Surgical therapy of vulvar cancer: how to choose the correct reconstruction? J Gynecol Oncol 2016;27(06):e60

10 Gentileschi S, Servillo M, Garganese G, et al. The lymphatic superficial circumflex iliac vessels deep branch perforator flap: A new preventive approach to lower limb lymphedema after groin dissection-preliminary evidence. Microsurgery 2017;37(06): 564-573

11 Yoshimatsu H, Iida T, Yamamoto T, Hayashi A. Superficial circumflex iliac artery-based iliac bone flap transfer for reconstruction of bony defects. J Reconstr Microsurg 2018;34(09):719-728

$12 \mathrm{Kim} \mathrm{JH}$, Kim KN, Yoon CS. Reconstruction of moderate-sized distal limb defects using a superthin superficial circumflex iliac artery perforator flap. J Reconstr Microsurg 2015;31(09):631-635

13 Hong JP, Sun SH, Ben-Nakhi M. Modified superficial circumflex iliac artery perforator flap and supermicrosurgery technique for lower extremity reconstruction: a new approach for moderatesized defects. Ann Plast Surg 2013;71(04):380-383

14 Smit JM, Klein S, Werker PMN. An overview of methods for vascular mapping in the planning of free flaps. JPlast Reconstr Aesthet Surg 2010;63(09):e674-e682

15 Tashiro K, Harima M, Kato M, et al. Preoperative color Doppler ultrasound assessment in planning of SCIP flaps. J Plast Reconstr Aesthet Surg 2015;68(07):979-983

16 Sidhoum N, Dast S, Perez S, Assaf N, Herlin C, Sinna R. [Superficial circumflex iliac artery perforator flap (scip flap): revival of the inguinal donor site?] Ann Chir Plast Esthet 2017;62(06):646-651

17 Jin S, He Y, Tian Z, Feng S, Zhang Y. Superficial circumflex iliac artery perforator flap aided by color Doppler sonography mapping for like-with-like buccal reconstruction. Oral Surg Oral Med Oral Pathol Oral Radiol 2015;119(02):170-176

18 Suh HSP, Jeong HH, Choi DH, Hong JPJP. Study of the medial superficial perforator of the superficial circumflex iliac artery perforator flap using computed tomographic angiography and surgical anatomy in 142 patients. Plast Reconstr Surg 2017;139 (03):738-748

19 He Y, Jin S, Tian Z, et al. Superficial circumflex iliac artery perforator flap's imaging, anatomy and clinical applications in oral maxillofacial reconstruction. JCraniomaxillofac Surg 2016; $44(03): 242-248$ 
20 Goh TLH, Park SW, Cho JY, Choi JW, Hong JP. The search for the ideal thin skin flap: superficial circumflex iliac artery perforator flap-a review of 210 cases. Plast Reconstr Surg 2015;135(02): 592-601

21 Hong JP, Choi DH, Suh H, et al. A new plane of elevation: the superficial fascial plane for perforator flap elevation. JReconstr Microsurg 2014;30(07):491-496

22 Iida T, Yoshimatsu H, Hara H, Mihara M, Koshima I. Reconstruction of large facial defects using a sensate superficial circumflex iliac perforator flap based on the lateral cutaneous branches of the intercostal nerves. Ann Plast Surg 2014;72 (03):328-331

23 Choi DH, Goh T, Cho JY, Hong JP. Thin superficial circumflex iliac artery perforator flap and supermicrosurgery technique for face reconstruction. JCraniofac Surg 2014;25(06): 2130-2133

24 Oh TS, Lee HS, Hong JP. Diabetic foot reconstruction using free flaps increases 5-year-survival rate. J Plast Reconstr Aesthet Surg 2013;66(02):243-250

25 Giacalone G, Yamamoto T, Hayashi A, et al. Lymphatic supermicrosurgery for the treatment of recurrent lymphocele and severe lymphorrhea. Microsurgery 2019;39(04):326-331

26 Ono S, Hayashi H, Ohi H, Ogawa R. Imaging studies for preoperative planning of perforator flaps: an overview. Clin Plast Surg 2017;44(01):21-30
27 Gentileschi S, Servillo M, De Bonis F, et al. Radioanatomical study of the pedicle of the superficial circumflex iliac perforator flap. J Reconstr Microsurg 2019;35(09):669-676

28 Debelmas A, Camuzard O, Aguilar P, Qassemyar Q. Reliability of color Doppler ultrasound imaging for the assessment of anterolateral thigh flap perforators: a prospective study of 30 perforators. Plast Reconstr Surg 2018;141(03):762-766

29 Feng S, Min P, Grassetti L, et al. A prospective head-to-head comparison of color Doppler ultrasound and computed tomographic angiography in the preoperative planning of lower extremity perforator flaps. Plast Reconstr Surg 2016;137(01): 335-347

30 Feng S, Xi W, Zhang Z, et al. A reappraisal of the surgical planning of the superficial circumflex iliac artery perforator flap. JPlast Reconstr Aesthet Surg 2017;70(04):469-477

31 Pereira N, Kufeke M, Parada L, et al. Augmented reality microsurgical planning with a smartphone (arm-ps): a dissection route map in your pocket. JPlast Reconstr Aesthet Surg 2019;72(05):759-762

$32 \mathrm{Su} \mathrm{W}$, Lu L, Lazzeri D, et al. Contrast-enhanced ultrasound combined with three-dimensional reconstruction in preoperative perforator flap planning. Plast Reconstr Surg 2013;131(01): 80-93

33 Ono S, Hayashi H, Ohi H, Ogawa R. Imaging studies for preoperative planning of perforator flaps: an overview. Clin Plast Surg 2017;44(01):21-30 